\title{
Simple test for quantum channel capacity
}

\author{
Marcin L. Nowakowski ${ }^{1}$ and Pawel Horodecki ${ }^{1 *}$ \\ ${ }^{1}$ Faculty of Applied Physics and Mathematics, Gdansk University of Technology, 80-952 Gdansk, Poland
}

\begin{abstract}
Basing on states and channels isomorphism we point out that semidefinite programming can be used as a quick test for nonzero one-way quantum channel capacity. This can be achieved by search of symmetric extensions of states isomorphic to a given quantum channel. With this method we provide examples of quantum channels that can lead to high entanglement transmission but still have zero one-way capacity, in particular, regions of symmetric extendibility for isotropic states in arbitrary dimensions are presented. Further we derive a new entanglement parameter based on (normalised) relative entropy distance to the set of states that have symmetric extensions and show explicitly the symmetric extension of isotropic states being the nearest to singlets in the set of symmetrically extendible states. The suitable regularisation of the parameter provides a new upper bound on one-way distillable entanglement.
\end{abstract}

PACS numbers: 03.67.-a, 03.67.Hk

\section{INTRODUCTION}

Quantum channels [1] are very important notion of quantum information theory [2]. It has been proven [1] time that there is a connection between entanglement distillation [3] and quantum channels capacities. No-cloning principle has been used to prove that for some region quantum depolarising channel has zero capacity even if does not destroy entanglement [4].

Following seminal work [5] and asymptotic analysis [6] that predicted limit formulas form conjectured hashing inequality [6] recently the latter have been proven [7, 8, 9], in particular the proof of hashing inequality has been provided [9]. On the other hand the connection between quantum channels and entanglement distillation [1] has been developed [6, 10] leading in particular to strong nonadditivity effects in case of more than one receiver [11]. On the other hand an interesting technique based on approximate quantum cloning was used in Ref. [4] to point out limit of depolarising qubit channel. This approach has been further extended in an elegant way to the case of Pauli channels [12] via assymetric cloning machines.

In the present approach we shall use the above techniques, exploiting also a general notion of symmetric extension of quantum state that was used recently efficiently applied with help of semidefinite programming to characterise quantum entanglement [13] 14] and states that admit local hidden variables models [15].

To be more specific, in this paper we develop qualitative equivalence between entanglement distillation and quantum channels theory showing in particular that:

(i) semidefinite programming can serve as a simple and quick test for nonzero one-way channel capacity via looking for symmetric extensions of the state $\varrho(\Lambda)$,

(ii) if normalised and regularised, the distance of given quantum state above to the set of symmetrically extendible states provides a new entanglement parameter that leads to upper bound on one-way distillable entanglement of the state.

To show that SDP can lead to interesting results we provide the family of quantum channels that allow for quite high entanglement transmission, however have one-way capacity zero due to existence of symmetric extension of the corresponding quantum state. The corresponding extensions are explicitly constructed.

\section{ONE-WAY DISTILLABLE ENTANGLEMENT}

Following the idea [4] developing restriction on qubit depolarising channel from approximate quantum cloning we shall utilise general notion of symmetric extensions of quantum state (see [13, 14, 15]) to provide a general rule and examples of channels with zero one-way capacity. We show now that every state $\rho_{A B}(\Lambda)$ which has a symmetric extension $\rho_{A B B^{\prime}}$ has special featured $D_{\rightarrow}$ and $Q_{\rightarrow}$ according to its quantum channel implied by Jamiolkowski isomorphism. The following observation that describes above reads:

* Electronic address: pawel@mif.pg.gda.pl 
Observation 1. If any bipartite state $\rho_{A B}$ has a symmetric extension $\rho_{A B B^{\prime}}$, so that $\rho_{A B B^{\prime}}=\rho_{A B^{\prime} B}$, then for the one-way distillable entanglement there holds: $D_{\rightarrow}\left(\varrho_{A B}\right)=0$.

Proof of the above theorem is immediate and follows from quantum entanglement monogamy (cf. [4, 12]). If Alice sends classical information to Bob and they distill singlet in the protocol then the state can not have symmetric extension since Bob's colleague, say Brigitte (corresponding to index B') could also receive the same message from Alice and finally share a singlet with Alice too. But Alice's particle can not be maximally entangled with two different particles at the some time (this is just the entanglement monogamy property). So a symmetrically extendible state can not have one-way distillable entanglement nonzero. Combining the above observation we get immediately

Observation 2. A sufficient condition for one-way quantum capacity of given quantum channel $\Lambda$ to vanish is symmetric extendibility of the state $\varrho(\Lambda)$ isomorphic to the channel.

As a special example of application of these observations we use below bipartite state $\rho_{A B}$ that is extendible for $F \leq \frac{1}{2}$, moreover, notice that in this range the state may be quite strong entangled.

$$
\rho_{A B}=\left(\begin{array}{ccccccccc}
\frac{F}{3} & 0 & 0 & 0 & \frac{F}{3} & 0 & 0 & 0 & \frac{F}{3} \\
0 & \frac{(1-F)}{3} & 0 & 0 & 0 & 0 & 0 & 0 & 0 \\
0 & 0 & 0 & 0 & 0 & 0 & 0 & 0 & 0 \\
0 & 0 & 0 & 0 & 0 & 0 & 0 & 0 & 0 \\
\frac{F}{3} & 0 & 0 & 0 & \frac{F}{3} & 0 & 0 & 0 & \frac{F}{3} \\
0 & 0 & 0 & 0 & 0 & 0 & 0 & 0 & 0 \\
0 & 0 & 0 & 0 & 0 & 0 & \frac{(1-F)}{3} & 0 & 0 \\
0 & 0 & 0 & 0 & 0 & 0 & 0 & \frac{(1-F)}{3} & 0 \\
\frac{F}{3} & 0 & 0 & 0 & \frac{F}{3} & 0 & 0 & 0 & \frac{F}{3}
\end{array}\right)
$$

Notice that filtering on Bob's side the state $\rho_{A B}$ and in general any such a state does not change the extendibility, what may be simply proved. Applying filtering with $W=\operatorname{diag}\left[1, \frac{1}{\sqrt{F}}, \frac{1}{\sqrt{2-F}}\right]$ we get a state $\widetilde{\rho}_{A B}$ and maximally mixed $\widetilde{\rho_{A}}$ on Alice's side

$$
\begin{gathered}
\widetilde{\rho}_{A B}=\frac{W \otimes I \rho_{A B} W^{\dagger} \otimes I}{\operatorname{Tr}\left\{W \otimes I \rho_{A B} W^{\dagger} \otimes I\right\}}, \widetilde{\rho}_{A}=\frac{I}{3} \\
\widetilde{\rho}_{A B}=\left(\begin{array}{ccccccccc}
\frac{F}{3} & 0 & 0 & 0 & \frac{\sqrt{F}}{3} & 0 & 0 & 0 & \frac{F}{3 \sqrt{2-F}} \\
0 & \frac{1-F}{3} & 0 & 0 & 0 & 0 & 0 & 0 & 0 \\
0 & 0 & 0 & 0 & 0 & 0 & 0 & 0 & 0 \\
0 & 0 & 0 & 0 & 0 & 0 & 0 & 0 & 0 \\
\frac{\sqrt{F}}{3} & 0 & 0 & 0 & \frac{1}{3} & 0 & 0 & 0 & \frac{\sqrt{F}}{3 \sqrt{2-F}} \\
0 & 0 & 0 & 0 & 0 & 0 & 0 & 0 & 0 \\
0 & 0 & 0 & 0 & 0 & 0 & \frac{1-F}{3(2-F)} & 0 & 0 \\
0 & 0 & 0 & 0 & 0 & 0 & 0 & \frac{1-F}{3(2-F)} & 0 \\
\frac{F}{3 \sqrt{2-F}} & 0 & 0 & 0 & \frac{\sqrt{F}}{3 \sqrt{2-F}} & 0 & 0 & 0 & \frac{F}{3(2-F)}
\end{array}\right)
\end{gathered}
$$

For any of the above state the extension can be found by means of linear optimisation with help of SEDUMI module [23]. We have found the extension of $\rho_{A B}$ very easily, in fact we have for $F \leq \frac{1}{2}$ the following spectral decomposition of the extension $\rho_{B A B}$ :

$$
\left\{\begin{array}{l}
\left|\varphi_{0}\right\rangle=|020\rangle \text { and } \lambda_{0}=\frac{1-F}{6} \\
\left|\varphi_{1}\right\rangle=|001\rangle+|100\rangle+|111\rangle+|122\rangle+|221\rangle \text { and } \lambda_{1}=\frac{F}{3} \\
\left|\varphi_{2}\right\rangle=|021\rangle \text { and } \lambda_{2}=\frac{1-2 F}{6} \\
\left|\varphi_{3}\right\rangle=|101\rangle \text { and } \lambda_{3}=\frac{1-2 F}{3} \\
\left|\varphi_{4}\right\rangle=|120\rangle \text { and } \lambda_{4}=\frac{1-F}{6} \\
\left|\varphi_{5}\right\rangle=|121\rangle \text { and } \lambda_{5}=\frac{1-2 F}{6}
\end{array}\right.
$$

where generally eigenvalues have to fulfil following conditions so that after tracing out Brigitte we obtain $\rho_{A B}$ :

$$
\left\{\begin{array}{l}
\lambda_{0}+\lambda_{4}=\frac{1-F}{3} \\
\lambda_{2}+\lambda_{5}=\frac{1-2 F}{3}
\end{array}\right.
$$


According to these constructions we may find another state $\rho_{B A B}$ that is nearest (in the set of states constructed on above eigenvectors $)$ to singlet in sense of fidelity $\left(\mathcal{F}=\left\langle\Psi_{+}\left|\rho_{A B}\right| \Psi_{+}\right\rangle\right)$of its local reduction $\rho_{A B}$ :

$$
\left\{\begin{array}{l}
\rho_{B A B}=\frac{1}{5}\left|\varphi_{1}\right\rangle\left\langle\varphi_{1}\right| \\
\rho_{A B}=\frac{3}{5} P_{+}+\frac{1}{5}|01\rangle\left\langle 01\left|+\frac{1}{5}\right| 21\right\rangle\langle 21|
\end{array}\right.
$$

As a generalization of such states we construct states extreme in the above sense for arbitrary dimension:

$$
\Upsilon=\frac{d}{2 d-1} P_{+}+\frac{1}{2 d-1} \sum_{i=1}^{d-1}|i \quad 0\rangle\langle i \quad 0|
$$

We state now the following question as a natural conclusion of above analysis:

Question: What is the maximal possible value of fidelity of $\rho$ that we may obtain from states for which $Q_{\rightarrow}=0$ ?

\section{UPPER BOUND ON $D \rightarrow$}

In this section we consider the distance of any state from the set of extendible states. Note that the set of extendible states is convex and compact, what can be obviously obtained from the extendibility of any convex combination of extendible states. Subsequently, we show that the set is closed under local operations and one-way classical communication (1-LOCC) in the following lemma:

Lemma III.1. The set $\mathcal{E}_{A B}$ of symmetrically extendible states is mapped under $1-L O C C$ for $\Lambda: B\left(\mathcal{H}_{A B}\right) \rightarrow B\left(\mathcal{H}_{\widetilde{A} \widetilde{B}}\right)$ into the set of symmetrically extendible states $\mathcal{E}_{\widetilde{A} \widetilde{B}}$.

Proof.

$$
\begin{array}{r}
\rho_{A B} \subset \mathcal{E}_{A B} \Rightarrow \exists_{\rho_{A B B^{\prime}}} \rho_{A B B^{\prime}}=\rho_{A B^{\prime} B} \wedge \operatorname{Tr}_{B^{\prime}} \rho_{A B B^{\prime}}=\rho_{A B} \\
\Rightarrow \operatorname{Tr}_{\widetilde{B^{\prime}}} \Lambda\left(\rho_{A B B^{\prime}}\right)=\rho_{\widetilde{A} \widetilde{B}} \subset \mathcal{E}_{\widetilde{A} \widetilde{B}}
\end{array}
$$

where

$$
\begin{aligned}
\Lambda\left(\rho_{A B B^{\prime}}\right)= & \sum_{i, j=1}^{K, L}\left(I_{2}^{\widetilde{A}} \otimes W_{j i}^{B \rightarrow \widetilde{B}} \otimes W_{j i}^{B^{\prime} \rightarrow \widetilde{B^{\prime}}}\right)\left(V_{i}^{A \rightarrow \widetilde{A}} \otimes I_{1}^{B} \otimes I_{1}^{B^{\prime}}\right) \rho_{A B B^{\prime}} \times \\
& \times\left(V_{i}^{A \rightarrow \widetilde{A} \dagger} \otimes I_{1}^{B} \otimes I_{1}^{B^{\prime}}\right)\left(I_{2}^{\widetilde{A}} \otimes W_{j i}^{B \rightarrow \widetilde{B} \dagger} \otimes W_{j i}^{B^{\prime} \rightarrow \widetilde{B^{\prime} \dagger}}\right)
\end{aligned}
$$

and operations acting on Bob's side are trace-preserving due to the necessity of non-breaking the property of extendibility.

For our analysis we define the measure of this distance based on the definition of relative entropy:

Definition III.2. Assume that a convex set $\mathcal{E}_{A B}$ is a set of extendible states, i.e.

$$
\mathcal{E}_{A B}=\left\{\sigma_{A B}: \exists_{A B B^{\prime} C} \sigma_{A B}=\sigma_{A B^{\prime}}=\operatorname{Tr}_{C B}\left[\left|\Psi_{A B B^{\prime} C}\right\rangle\left\langle\Psi_{A B B^{\prime} C}\right|\right]\right\}
$$

Then the distance of a state $\rho_{A B}$ on $\mathcal{H}_{A B}=\mathcal{H}_{A} \otimes \mathcal{H}_{B}$ with $\operatorname{dim} \mathcal{H}_{A}=d_{A}$ and $\operatorname{dim} \mathcal{H}_{B}=d_{B}$ from the set of extendible states $\mathcal{E}_{A B}$ of $d \otimes d$ type where $d=\max \left[d_{A}, d_{B}\right]$ is defined by

$$
R_{\mathcal{E}_{A B}}\left(\rho_{A B}\right)=\delta_{A B} \inf _{\sigma_{A B} \in \mathcal{E}} R\left(\widetilde{\rho}_{A B} \| \sigma_{A B}\right)
$$

where $\forall_{\rho, \sigma} R(\rho \| \sigma)=\operatorname{Tr}[\rho \log \rho-\rho \log \sigma]$ and $\delta=-\frac{\log d}{\log \frac{(d+1)}{2 d}}$ with $d=\max \left[d_{A}, d_{B}\right]$ due to normalization of this function on maximally entangled states. In the formula (9) $\widetilde{\rho}_{A B}$ is taken as a state of $d \otimes d$ type (after embedding $\rho_{A B}$ into $d \otimes d$ space).

Using techniques [26] we show that the nearest one in arbitrary dimension is a state $\rho\left(d, F_{\max }\right)$ from subset of isotropic states $\rho(d, F)$ [27] with fidelity $F \leq F_{\max }$ for which those are symmetrically extendible:

$$
F_{\max }=\frac{d+1}{2 d}
$$




$$
\rho(d, F)=\frac{d^{2}}{d^{2}-1}\left[(1-F) \frac{I}{d^{2}}+\left(F-\frac{1}{d^{2}}\right) P_{+}\right]
$$

Indeed, following [26] one needs to analyze operators from a six dimensional non-commutative $\mathbf{C}^{*}$-algebra that are $\bar{U} \otimes U \otimes U$-invariant and $V_{(23)}$-invariant. Such operators $S$ will be represented as a linear combination of the basis elements of the algebra: $B=\left\{S_{+}, S_{-}, S_{0}, S_{1}, S_{2}, S_{3}\right\}$ where for the trace condition one obtains [26] conditions for factors of the combination: $s_{2}=s_{3}=0$ and, further, from positivity: $s_{0}=1-s_{+}-s_{-}$.

$$
S=s_{+} S_{+}+s_{-} S_{-}+s_{0} S_{0}+s_{1} S_{1}
$$

The matter of interest is now the tetrahedron in three-dimensional euclidian space of parameters $\left(s_{+}, s_{-}, s_{1}\right)$ confined by the hyperplanes [26]: $\left\{h_{1}^{\prime}, h_{2}^{\prime}, h_{3}^{\prime}, h_{4}^{\prime}\right\}$ in which exists the state $\Omega_{A B E}$ giving the searched symmetrically extendible reduction $\rho_{A B}$. For maximizing the distance of the unknown state $\rho_{A B}$ to singlet it suffices to find the maximization over fidelity $\widetilde{F}$ between the symmetric extension represented as $\Omega_{A B E}$ and virtually extended unnormalized operator $\rho_{A B B^{\prime}}=P_{+} \otimes I$ as $\widetilde{F}_{\text {max }}=\operatorname{Tr}\left[P_{+} \otimes I \Omega_{A B E}\right]=\operatorname{Tr}\left[P_{+} \rho_{A B}\right]=F_{\max }:$

$$
\begin{gathered}
\left\{\begin{array}{l}
F_{+}=\operatorname{Tr}\left[\left(P_{+} \otimes I\right) S_{+}\right] / \operatorname{Tr}\left[S_{+}^{2}\right]=0 \\
F_{-}=\operatorname{Tr}\left[\left(P_{+} \otimes I\right) S_{-}\right]=0 \\
F_{0}=\operatorname{Tr}\left[\left(P_{+} \otimes I\right) S_{0}\right] / \operatorname{Tr}\left[S_{0}^{2}\right]=d / 2 d \\
F_{1}=\operatorname{Tr}\left[\left(P_{+} \otimes I\right) S_{1}\right] / \operatorname{Tr}\left[S_{1}^{2}\right]=1 / 2 d
\end{array}\right. \\
\left\{\begin{array}{l}
\widetilde{F}=F_{0}+\vec{s} \circ \vec{f} \\
\widetilde{F}_{\max }=\max _{\vec{s} \in \Delta} \widetilde{F}
\end{array}\right.
\end{gathered}
$$

where $\Delta$ denotes the tetrahedron bounded by mentioned hyperplanes, $\vec{f}=\left[F_{+}-F_{0}, F_{-}-F_{0}, F_{1}\right]$ and $\vec{s}=\left[s_{+}, s_{-}, s_{0}\right]$. Normalization of parameters $F_{i}$ inherits from the commutation relations [26] between operators $S_{i}$. Maximization results in $\vec{s}=[0,0,1]$ that relates to the found aforementioned isotropic states $\rho_{A B}=\rho\left(d, F_{\max }\right)$. The explicit form of the tripartite symmetric extension of isotropic states $\rho\left(d, F_{\max }\right)$ in the border of extendibility is:

$$
\Omega_{A B E}=\frac{1}{2 d}\left(S_{0}+S_{1}\right)
$$

where [26]:

$$
S_{0}=\frac{1}{d^{2}-1}(d(X+V X V)-(X V+V X)) \text { and } S_{1}=\frac{1}{d^{2}-1}(d(X V+V X)-(X+V X V))
$$

for

$$
|\Phi\rangle=\sum_{i}|i i\rangle, X=|\Phi\rangle\left\langle\Phi\left|\otimes I, V=V_{(23)}=\sum_{i j k}\right| i j k\right\rangle\langle i k j| .
$$

It is important to notice that the same results can be obtained numerically by means of linear programming methods that we have utilized to find the broad class of symmetrically extendible states.

Following we analyze if similarly to distance from separable states one can construct an appropriate entanglement measure basing on (9). The normalized distance from the set of extendible states does not satisfy though all necessary conditions [24, 25] that every measure of one-way distillable entanglement has to satisfy: introduction of the normalization factor $\delta_{A B}$ causes that $R_{\mathcal{E}_{A B}}(\rho)$ becomes explicitly dependant on the dimension of the system $A B$, therefore, for protocols increasing dimension of input state the parameter is not a monotone:

A1. If $\sigma_{A B}$ is separable then $R_{\mathcal{E}_{A B}}\left(\sigma_{A B}\right)=0$ due to the fact that every separable state is extendible.

A2. Local unitary operations leave $R_{\mathcal{E}_{A B}}\left(\sigma_{A B}\right)$ invariant, that is satisfied due to invariancy of distance measures under local unitary transformations, i.e. $R_{\mathcal{E}_{A B}}\left(\sigma_{A B}\right)=R_{\mathcal{E}_{A B}}\left(U_{A} \otimes U_{B} \sigma_{A B} U_{A}^{\dagger} \otimes U_{B}^{\dagger}\right)$.

A3. (Restricted 1-LOCC monotonicity.) The parameter $R_{\mathcal{E}_{A B}}\left(\sigma_{A B}\right)$ of one-way distillable entanglement does not increase under non-increasing dimension 1-LOCC, i.e. $\Lambda: B\left(\mathcal{H}_{A B}\right) \rightarrow B\left(\mathcal{H}_{\widetilde{A} \widetilde{B}}\right)$ with $n_{A B}=\max \left[d_{A}, d_{B}\right]$, $n_{\widetilde{A} \widetilde{B}}=\max \left[d_{\widetilde{A}}, d_{\widetilde{B}}\right]$ for $n_{A B} \geq n_{\widetilde{A} \widetilde{B}}$, then

$$
R_{\mathcal{E}_{\tilde{A} \widetilde{B}}}\left(\Lambda \sigma_{A B}\right) \leq R_{\mathcal{E}_{A B}}\left(\sigma_{A B}\right)
$$


This condition may be simply proved due to non-increasing of $R(\rho \| \sigma)$ under a subclass of 1-LOCC operations $\Lambda$ that is stated above in the lemma. Namely, because $\Lambda \mathcal{E}_{A B} \subset \mathcal{E}_{\widetilde{A} \widetilde{B}}$ and assuming that $\sigma^{*}$ is an extendible state that realizes the minimal value in eq.(9) we have:

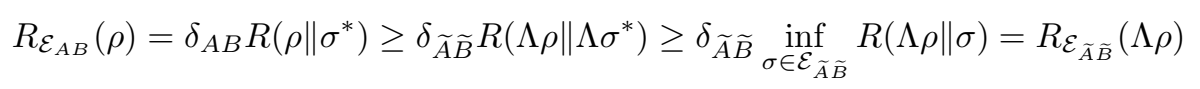

where $n_{A B} \geq n_{\widetilde{A} \widetilde{B}}$ derives the condition $\delta_{A B} \geq \delta_{\widetilde{A} \widetilde{B}}$.

However, we show further that the entanglement parameter can be utilized for bounding one-way entanglement of distillation due to preparation of the measure in asymptotic regime.

In general, every entanglement parameter of type $E(\sigma)=\alpha \inf _{\rho \in \Delta} \mathcal{D}(\sigma \| \rho)$ where $\mathcal{D}(\sigma \| \rho)$ is appropriate distance between $\sigma$ and $\rho, \Delta$ denotes the characteristic set to which the distance is measured and $\alpha$ normalizes the parameter so that $E\left(\left|\Psi_{+}\right\rangle\left\langle\Psi_{+}\right|\right)=\log d$ is not monotonic, i.e. $\exists_{\Lambda} E(\sigma)>E(\Lambda(\sigma))$. For $R_{\mathcal{E}_{A B}}$ unitary injection of input state $\rho_{A B}$ into higher dimensional space gives $R_{\mathcal{E}_{A B}}(\rho)>R_{\mathcal{E}_{\tilde{A} \tilde{B}}}(\Lambda(\rho))$.

Additionally, following analysis in [28, 29], we show that the entanglement parameter satisfies:

B1. (Continuity on isotropic states.) We may simply show that this parameter is continuous on isotropic states $\rho\left(d_{n}, F_{n}\right)$ with $F_{n} \rightarrow 1, d_{n} \rightarrow \infty$ that means

$$
\frac{R_{\mathcal{E}}\left(\rho\left(d_{n}, F_{n}\right)\right)}{\log d_{n}} \rightarrow 1
$$

as then $R_{\mathcal{E}}\left(\rho\left(d_{n}, F_{n}\right)\right) \rightarrow \log d_{n}$ that is easy to check.

Following the papers [28, 30] and the above definition we define the distance in asymptotic regime as follows:

$$
R_{\mathcal{E}_{A B}}^{\infty}\left(\rho_{A B}\right)=\limsup _{n \rightarrow \infty} \frac{R_{\mathcal{E}_{A B}}\left(\rho_{A B}{ }^{\otimes n}\right)}{n}
$$

One can also propose other measures, but this will be subject of analysis elsewhere [31].

Having defined above regularized parameter $R_{\mathcal{E}_{A B}}^{\infty}\left(\rho_{A B}\right)$, we are able now to determine the upper bound on the one-way distillable entanglement. In [9] Devetak and Winter have proved very powerful conjecture called "hashing inequality"

$$
D_{\rightarrow} \geq S\left(\rho_{B}\right)-S\left(\rho_{A B}\right)
$$

from which one may find particular states of non-zero $D_{\rightarrow}$. For the very features of measures that bound the distillable entanglement $D_{\rightarrow}$, defined in [28, 29], where was shown that monotonicity, continuity on isotropic states are sufficient for any properly regularised function to be upper bound for $D_{\rightarrow}$, we may prove now the following theorem exploiting only distillation protocols in the line of the proof:

Theorem III.3. For any bipartite state $\rho_{A B}$ there holds:

$$
D_{\rightarrow}\left(\rho_{A B}\right) \leq R_{\mathcal{E}_{A B}}^{\infty}\left(\rho_{A B}\right)
$$

Proof. Any one-way distillation protocol can be reduced to the distillation protocol [28, 29, 30] where the input is $\rho^{\otimes n}$ and the output is a family of the states $\rho\left(d_{n}, F_{n}\right)$ with $\lim _{n \rightarrow \infty} \frac{\log d_{n}}{n}=D_{\rightarrow}(\rho)$ and $F_{n} \rightarrow 1$. We may always put $d_{n} \leq n_{A B}^{n}$ for $n_{A B}=\min \left[d_{A}, d_{B}\right]$ since there holds $D_{\rightarrow}(\rho) \leq \log n_{A B}$. Thus, we can consider only 1-LOCC nonincreasing dimensions of input and so monotonicity of $R_{\mathcal{E}_{A B}}$ holds. By analogy with the theorem put in [28, 29, 30] the properties (A3) and (B1) imply that $R_{\mathcal{E}_{A B}}^{\infty}\left(\rho_{A B}\right)$ is upper bound for $D_{\rightarrow}$. The regularisation (18) with supreme value enables upper bound of $D_{\rightarrow}$.

\section{CONCLUSIONS}

Quantum channels theory still has many unsolved problems. We have pointed out a general test for zero capacity of one-way channel capacity (which has been shown to be equal to zero-way capacity [18]). The test is based on checking of the existence of symmetric extension of a state isomorphic to a given channel. The test can be very easily performed with help of popular semi-definite programming codes. Finally, basing on the test, we have found a new parameter of entanglement. Its suitable regularized version is an upper bound on one-way distillable entanglement of given quantum state. Note that, although the entanglement monogamy property was known for a long time, 
this is the first entanglement parameter basing explicitly on that property and the symmetric extension of quantum states. We hope that the above results will help in further analysis of various aspects of quantum channels. It is very interesting that recently developed complete hierarchies approach to separability problem 34] has been extended [33] to include symmetric extensions of quantum operators, which leads to class of entanglement measures. This gives a hope that symmetric extensions will be a useful tool not only to qualify but also to quantify some aspects of quantum entanglement.

[1] C. H. Bennett, D. P. DiVincenzo, J. A. Smolin and W. K. Wootters Phys. Rev. A 5, 3824 (1996)

[2] H.-K. Lo, S. Popescu and T. Spiller, (eds.) Introduction in quantum information and computation, World Scientific, (1998); J. Gruska, Quantum Computing, McGraw-Hill, London 1999; D. Bouwmeester, A. K. Ekert, A. Zeilinger (eds.), The physics of quantum information : quantum cryptography, quantum teleportation, quantum computation; Springer, New York 2000; M. A. Nielsen and I. L. Chuang, Quantum Computation and Quantum Information, Cambridge University Press, Cambridge 2000; G. Alber, T. Beth, M. Horodecki, P. Horodecki, R. Horodecki, M. Röttler, H. Weinfurter, R. F. Werner, and A. Zeilinger, Quantum information: an introduction to basic theoretical concepts and experiments., volume 173 of Springer Tracts in Modern Physics, Springer, Berlin 2001.

[3] C. H. Bennett, G. Brassard, S. Popescu, B. Schumacher, J. A. Smolin and W. K. Wootters, Phys.Rev.Lett. 76, 722 (1996).

[4] D. Bruss, D. P. DiVincenzo, A. Ekert, C. A. Fuchs, C. Macchiavello and J. A. Smolin, Phys. Rev. A, 57, 2368 (1998).

[5] H. Barnum, E. Knill and M. A. Nielsen, IEEE Trans.Info.Theor. 461317 (2000).

[6] M. Horodecki, P. Horodecki and R.Horodecki, Phys. Rev. Lett. 85, 433 (2000).

[7] P. W. Shor, Capacities of quantum channels and how to find them, Preprint quant-ph/0304102.

[8] I. Devetak I, The private classical information capacity and quantum information capacity of a quantum channel, Preprint quant-ph/0304127

[9] I. Devetak and A. Winter, Phys. Rev. Lett. 93, 080501 (2004).

[10] P. Horodecki, Centr. Eur. J. Phys. 4, 695 (2003).

[11] W. Dür, J. I. Cirac and P. Horodecki, Phys. Rev. Lett. 93, 020503 (2004).

[12] N. J. Cerf, Phys. Rev. Lett. 84, 4497 (2000).

[13] A. C. Doherty, P. A. Parillo and F. M. Spedalieri, Phys. Rev. Lett. 88, 187904 (2002) .

[14] A. C. Doherty, P. A. Parillo and F. M. Spedalieri, Phys. Rev. A 69, 022308 (2004).

[15] B. M. Terhal, A. C. Doherty and D. Schwab, Phys. Rev. Lett. 90, (2003).

[16] A. Jamiolkowski, Rep. Math. Phys. 3, 275 (1972).

[17] M. D. Choi, Linear Algebra and Its Applications 10, 285 (1975).

[18] H. Barnum, J. A. Smolin and B. M. Terhal, Phys. Rev. A 58, 3496 (1998).

[19] C. H. Bennett, D. P. DiVincenzo, and J. A. Smolin, Phys. Rev. Lett. 78, 3217 (1997).

[20] G. Vidal, On the continuity of asymptotic measures of entanglement, Preprint quant-ph/0203107

[21] G. Brassard, P. Horodecki and T. Mor, IBM J. Res. Dev. 84, 87 (2004).

[22] D. Kretschmann and R. F. Werner, New J. Phys. 626 (2004).

[23] J. Sturm, SEDUMI VERSION 1.05.2001, http://fewcal.kub.nl/sturm/software/sedumi.html

[24] V. Vedral, M. B. Plenio, M. A. Rippin and P. L. Knight, Phys. Rev. Lett. 78, 2275 (1997).

[25] V. Vedral and M. B. Plenio, Phys. Rev. A 57, 1619 (1998).

[26] T. Eggeling, R. F. Werner, Phys. Rev. A 63, 042111 (2001).

[27] M. Horodecki and P. Horodecki, Phys. Rev. A 59, 4206 (1999).

[28] M. Horodecki, Quant. Info. Comp. 1, 1 (2001).

[29] M. J. Donald, M. Horodecki and O. Rudolph, J. Math. Phys. 43, 4252 (2002).

[30] M. Horodecki, P. Horodecki and R.Horodecki, Phys. Rev. Lett. 84, 2014 (2000).

[31] For instance one can propose fidelity of state according to the nearest purified extension as follows $F_{\mathcal{E}}\left(\rho_{A B}\right)=$ $\inf _{\sigma_{A B} \in \mathcal{E}} F\left(\rho_{A B}, \sigma_{A B}\right)=\inf _{\sigma_{A B} \in \mathcal{E}}\left|\left\langle\Phi_{A B B^{\prime} C} \mid \Psi_{A B B^{\prime} C}\right\rangle\right|$ where the set $\mathcal{E}$ is defined as in above definition and both $\Phi_{A B B^{\prime} C}$ and $\Psi_{A B B^{\prime} C}$ is a purification of a suitable state. It can be shown that such quantity is also restricted 1-LOCC monotone.

[32] M. J. Donald and M. Horodecki, Phys. Lett. A 264, 257 (1999).

[33] J. Eisert, private communication.

[34] J. Eisert, P. Hyllus, O. Guehne and M. Curty, Phys. Rev. A 70, 062317 (2004). 